\title{
Narrative review of the role of artificial intelligence to improve aortic valve disease management
}

\author{
Martin Thoenes ${ }^{1}$, Anurag Agarwal ${ }^{2}$, David Grundmann ${ }^{3}$, Carmen Ferrero ${ }^{4}$, Andrew McDonald ${ }^{2}$, \\ Peter Bramlage $^{5}$, Richard P. Steeds ${ }^{6}$
}

${ }^{1}$ Léman Research Institute, Schaffhausen am Rheinfall, Switerzland; ${ }^{2}$ Department of Engineering, University of Cambridge, UK; ${ }^{3}$ Cardiology Department, UKE Hamburg, Germany; ${ }^{4}$ Departamento de Farmacia y Tecnología Farmacéutica, Facultad de Farmacia, Universidad de Sevilla, Spain; ${ }^{5}$ Institute for Pharmacology and Preventive Medicine, Cloppenburg, Germany; ${ }^{6}$ Queen Elizabeth Hospital \& Institute of Cardiovascular Sciences, University of Birmingham, Birmingham, UK

Contributions: (I) Conception and design: M Thoenes, P Bramlage; (II) Administrative support: None; (III) Provision of study materials or patients: None; (IV) Collection and assembly of data: None; (V) Data analysis and interpretation: M Thoenes, P Bramlage, RP Steeds; (VI) Manuscript writing: All authors; (VII) Final approval of manuscript: All authors.

Correspondence to: Martin Thoenes, MD. Léman Research Institute, Berbiceweg 5, 8312 Neuhausen am Rheinfall, Switzerland.

Email: martin.thoenes@leman-research.ch.

\begin{abstract}
Valvular heart disease (VHD) is a chronic progressive condition with an increasing prevalence in the Western world due to aging populations. VHD is often diagnosed at a late stage when patients are symptomatic and the outcomes of therapy, including valve replacement, may be sub-optimal due the development of secondary complications, including left ventricular (LV) dysfunction. The clinical application of artificial intelligence (AI), including machine learning (ML), has promise in supporting not only early and more timely diagnosis, but also hastening patient referral and ensuring optimal treatment of VHD. As physician auscultation lacks accuracy in diagnosis of significant VHD, computer-aided auscultation (CAA) with the help of a commercially available digital stethoscopes improves the detection and classification of heart murmurs. Although used little in current clinical practice, CAA can screen large populations at low cost with high accuracy for VHD and faciliate appropriate patient referral. Echocardiography remains the next step in assessment and planning management and AI is delivering major changes in speeding training, improving image quality by pattern recognition and image sorting, as well as automated measurement of multiple variables, thereby improving accuracy. Furthermore, AI then has the potential to hasten patient disposal, by automated alerts for red-flag findings, as well as decision support in dealing with results. In management, there is great potential in ML-enabled tools to support comprehensive disease monitoring and individualized treatment decisions. Using data from multiple sources, including demographic and clinical risk data to image variables and electronic reports from electronic medical records, specific patient phenotypes may be identified that are associated with greater risk or modeled to the estimate trajectory of VHD progression. Finally, AI algorithms are of proven value in planning intervention, facilitating transcatheter valve replacement by automated measurements of anatomical dimensions derived from imaging data to improve valve selection, valve size and method of delivery.
\end{abstract}

Keywords: Valvular heart disease (VHD); artificial intelligence (AI); auscultation

Submitted May 04, 2020. Accepted for publication Nov 05, 2020.

doi: $10.21037 /$ jtd-20-1837

View this article at: http://dx.doi.org/10.21037/jtd-20-1837

(c) Journal of Thoracic Disease. All rights reserved. 


\section{Introduction}

Valvular heart disease (VHD) involves disruption of the anatomic integrity of one or more heart valves causing valvular stenosis, valvular regurgitation or a combination of the two. VHD is usually considered either as primary, in which there are inherited or acquired abnormalities of the valve apparatus itself (for example bicuspid aortic valve or calcific aortic stenosis, respectively), or secondary, in which case valve function fails either due to myocardial dysfunction (heart failure) with abnormal mechanical stress and distortion of the valvular/subvalvular apparatus (mitral or tricuspid regurgitation) or due to aortic root disease and dilation (aortic regurgitation) (1). In the Western world, degenerative aortic stenosis and mitral regurgitation are the most common forms of VHD, with increasing prevalence as the population ages (2-4). The financial burden to health systems is huge, not only in terms of the cost of monitoring and treatment where feasible but also the slow progression to dependence of those patients exposed to the chronic onset of VHD.

In contrast to natural intelligence displayed by humans, artificial intelligence (AI) describes intelligence demonstrated by any device that perceives its environment and takes actions thereby mimicking human cognitive functions, such as learning and problem solving. Machine learning (ML) is a subfield of AI, where a mathematical model is designed and trained to make predictions using a large dataset. Due to advances in algorithmic design and the increasing availability, amount and complexity of data ("big data"), the role of ML has grown in the healthcare sector to facilitate diagnosis and to recommend treatments based on risk-benefit analysis. This has advantages, not only in earlier diagnosis, improvement in reliability of testing, and reduction in human errors due to cognitive bias, but also by supporting patient engagement and speeding administration through automatization (5). A promising application of $\mathrm{AI}$ analytics in healthcare involves precision medicine for patients with chronic disease with variable progression rates and unpredictable events, e.g., diabetes, in order to optimize individual prognostication and treatment (6). Over the past decade, the use of ML has also grown in cardiovascular medicine to improve quality of care, cost-effectiveness and mortality in patients with heterogenous diseases, such as heart failure, cardiomyopathy, arterial/pulmonary hypertension and coronary artery disease (7). More recently, publications have focused on the application of $\mathrm{AI}$ in $\mathrm{VHD}$ for detecting mitral valve disease/regurgitation and tricuspid valve disease (8-10). This article provides insights into the application of AI analytics in the management of patients with VHD, with a particular focus on those patients with aortic stenosis (AS) (Figure 1).

We present the following article in accordance with the Narrative Review reporting checklist (available at http:// dx.doi.org/10.21037/jtd-20-1837).

\section{Al in VHD}

\section{Auscultation}

VHD is most often a chronic progressive condition with an insidious onset that is clinically silent for many years before diagnosis, but then requires intervention which may be curative, although more often requires continued long-term monitoring (Figure 2) (11). Sclerosis of the aortic valve can be detected early in life, followed by progressive calcification and thickening at a variable pace, ultimately leading to clinical AS with characteristic hemodynamic alterations at which stage the patient may develop symptoms or present with heart failure (12). Data over many years has repeatedly shown that most patients are diagnosed at a late stage when symptomatic or suffering from complications, such as LV dysfunction (3). Factors contributing to late diagnosis of VHD include a low level of patient awareness, the underuse of cardiac auscultation, and a low skill-base for auscultation among both primary and secondary care physicians $(13,14)$. Physician auscultation lacks both sensitivity (up to $43 \%$ ) and specificity (69\%) for diagnosing significant VHD even when the clinicians are experienced (15). Heart sounds are variable in frequency, intensity, timing, location and are affected by other noise signals from the chest and upper abdomen, so can be challenging to interpret. Digital stethoscopes improve the detection of murmurs by converting an acoustic sound to an electronic signal, which can be further amplified, filtered and digitalized (16).

Development of computer-aided auscultation (CAA) algorithms has been limited by the availability of high-quality heart sound recordings, which are needed to train an accurate ML system. The vast majority of stethoscopes in use are analogue, so heart sounds are not generally recorded electronically as part of the patient record. Collection of heart sound recordings, therefore, requires specific research studies. Automated classification of heart sounds has previously been attempted by many research groups, though many of these earlier studies are flawed due to the use of small datasets, 


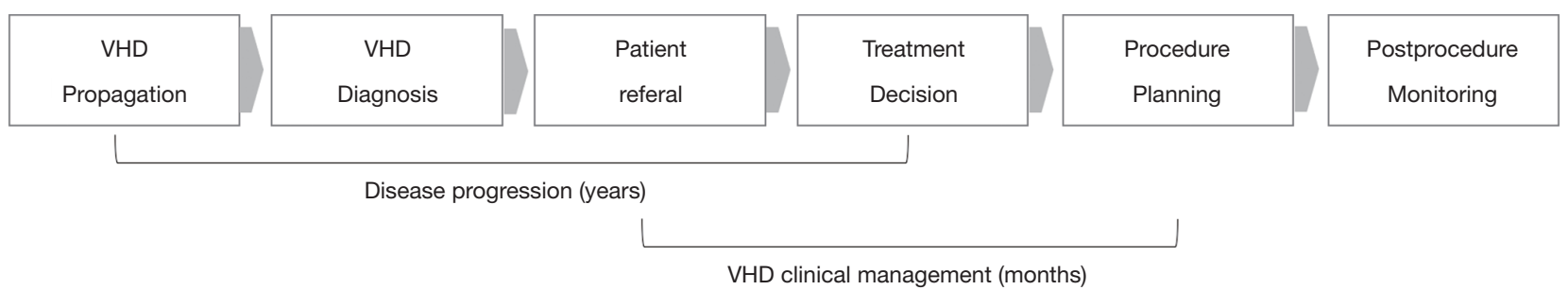

Figure 1 VHD—disease management pathway.

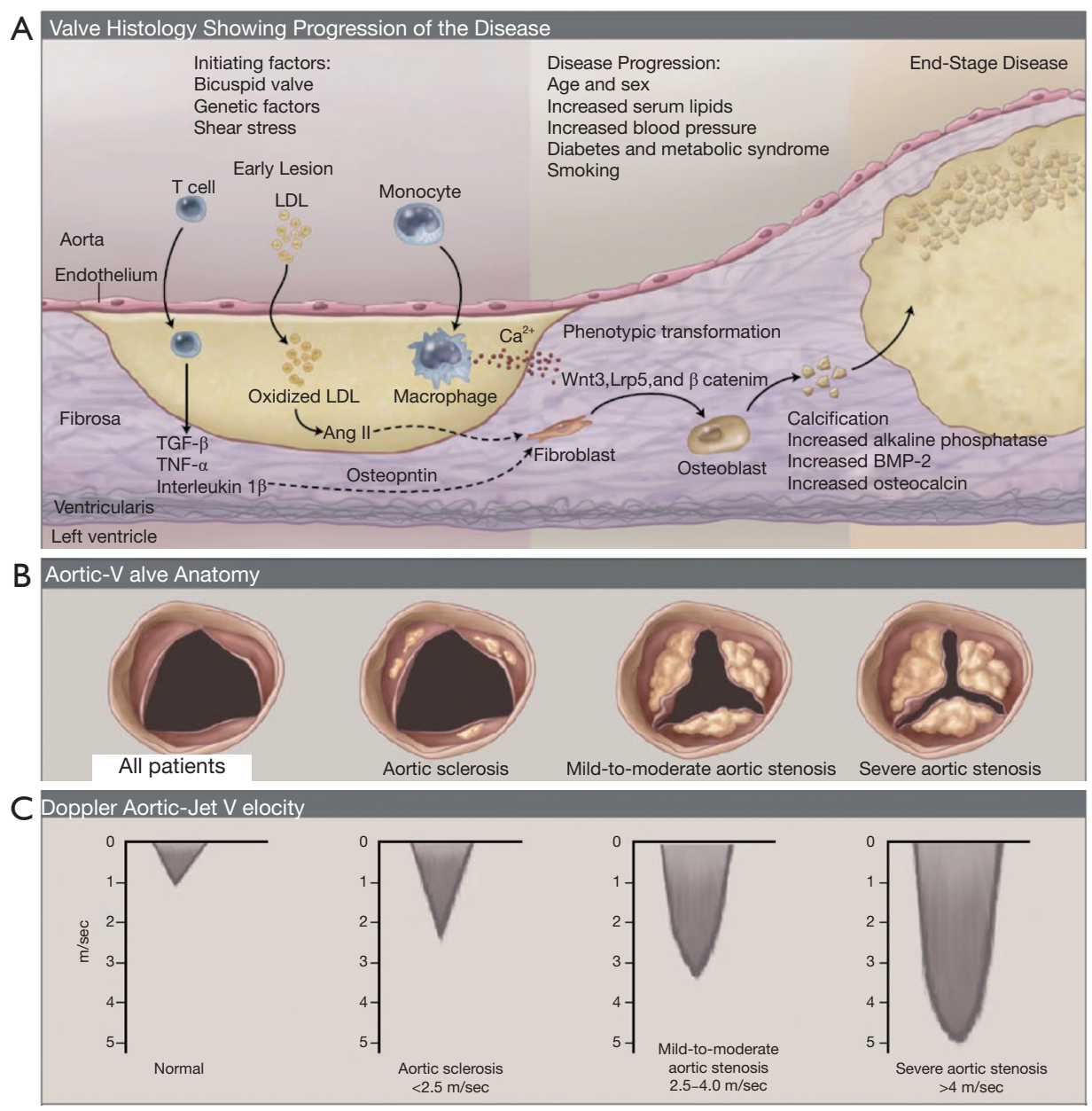

Figure 2 Calcific aortic stenosis-a chronic progressive condition (11). (A) The histology of the early lesion is characterized by a subendothelial accumulation of oxidized low-density lipoprotein (LDL), production of angiotensin (Ang) II, and inflammation with T lymphocytes and macrophages. Disease progression occurs by several mechanisms, including local production of proteins, such as osteopontin, osteocalcin, and bone morphogenic protein 2 (BMP-2), which mediate tissue calcification; activation of inflammatory signaling pathways, including tumor necrosis factor $\alpha$ (TNF- $\alpha$ ), tumor growth factor $\beta$ (TGF- $\beta$ ), the complement system, C-reactive protein, and interleukin-1 $\beta$; and changes in tissue matrix, including the accumulation of tenascin $\mathrm{C}$, and up-regulation of matrix metalloproteinase 2 and alkaline phosphatase activity. In addition, leaflet fibroblasts undergo phenotypic transformation into osteoblasts, regulated by the Wnt3-Lrp5- $\beta$ catenin signaling pathway. Microscopic accumulations of extracellular calcification $\left(\mathrm{Ca}^{2+}\right)$ are present early in the disease process, with progressive calcification as the disease progresses and areas of frank bone formation in end-stage disease. The corresponding changes in aortic-valve anatomy are viewed from the aortic side with the valve open in systole (B) and in Doppler aortic-jet velocity (C). 
Table $1 \mathrm{AI}$ in VHD: Auscultation

\begin{tabular}{ll}
\hline Advantages of Al stethoscopes & Limitations of Al stethoscopes \\
\hline More accurate than doctors using analogue stethoscopes & $\begin{array}{l}\text { Not 100\% accurate. The sensitivity and specificity should be factored in } \\
\text { healthcare decisions. Algorithm tend to be sensitive to the type of } \\
\text { stethoscope and quality and range of data }\end{array}$ \\
$\begin{array}{ll}\text { Can lead to better screening and reduce over-referrals for echo } \\
\text { Rery quick and cheap compared to echocardiography }\end{array}$ & $\begin{array}{l}\text { Ret are not always interpretable. The stethoscope will give a binary } \\
\text { decision but not give the physical reasoning for it }\end{array}$ \\
$\begin{array}{ll}\text { Someone without medical training could use it } & \text { stethoscopes }\end{array}$ \\
$\begin{array}{ll}\text { Help with telemedicine and have consistent patient records for } \\
\text { tracking and monitoring. Easy to share data with consultants }\end{array}$ & $\begin{array}{l}\text { There could be data privacy issues if the recordings are stored on a } \\
\text { that require careful placement of the stethoscope on the chest }\end{array}$ \\
\hline
\end{tabular}

unrealistically clean recordings, or lack of a separate test set for evaluation. The PhysioNet/Computing in Cardiology Challenge 2016 (17) attempted to address these issues by providing a large, varied database of 3,153 recordings, assembled from seven research groups. The challenge asked researchers to build an algorithm to classify single recordings as 'abnormal' or 'normal', and then evaluated their designs on a withheld test set. The winner of the challenge achieved a sensitivity of $94 \%$ and a specificity of $78 \%$ (18). The growing size and quality of datasets has enabled encouraging CAA results, though reported results can vary widely depending on the make-up of the test dataset used. Using the John Hopkins Cardiac Auscultatory Recording Database (CARD), Thompson et al. conducted a virtual clinical trial of CSD Labs' 'eMurmur' algorithm on 3,180 heart sounds from 603 outpatients. Sensitivity, specificity, and accuracy for detection of pathologic conditions were 93\% (CI: 90-95\%), 81\% (CI: $7-85 \%$ ) and $88 \%$ (CI: 85-91\%), respectively (19). However, data deemed to be 'noisy' or lacking an audible murmur was removed before testing, which will artificially improve the algorithm's performance compared to real-life use. In another pilot trial conducted in a pediatric cardiology setting, phonocardiograms of 106 patients with either no murmur, innocent murmurs or pathological murmurs were analyzed with a computerized algorithm and accuracy in detection of murmurs was compared with interpreted echocardiograms as a gold standard. The test algorithm was able to detect murmurs with a sensitivity of $87 \%$, specificity of $100 \%$, a positive predictive value of $100 \%$ (negative predictive value $90 \%$ ), and $94 \%$ accuracy (20). Improved sensitivity of CAA compared to physician auscultation to detect positive echocardiography findings was also confirmed in neonates (21). Furthermore, CAA appears to be a relevant decision support tool not only for the detection of pathological murmurs but also appropriate referral of patients for further evaluation as shown by Watrous et al. (22) (referral sensitivity of $93 \%$, specificity $79 \%$ ).

The application of AI-analytics has been the foundation for the development of commercially available, mobile AI-enabled stethoscopes (e.g., Stethee ${ }^{\mathrm{TM}}$, M3DICINE, Brisbane, AUS; StethoMe ${ }^{\mathrm{TM}}$, Poznan, and PL), as well as FDA-approved software applications that can be used with a smartphone or personal computer and a digital stethoscope for automated detection of heart murmurs (Table 1). Available technology enables automated heart rate detection based on an audiosignal, followed by heart sound segmentation (systole/diastole), and feature extraction with subsequent classification by using ML approaches to construct a classifier based on training data. There is considerable variation in autonomy of currently available analysis algorithms, with systems operating fully autonomously (eMurmur ID, Ottawa, CAN), or applications requiring user interaction and data interpretation (Zargis Cardioscan $^{\mathrm{TM}}$, Princeton, USA; SensiCardiac ${ }^{\mathrm{TM}}$, Diacoustic Medical Devices, Stellenbosch, South Africa).

Despite the commercial availability of decision support tools to facilitate the diagnosis of VHD with cardiac auscultation, the application of CAA in daily clinical practice appears to be limited and its utility has been mostly investigated in the pediatric setting. The results in pediatric populations may well be less reproducible than in adult populations in whom obesity and other co-morbidities, such as chronic lung disease, may interfere with acoustic quality. CAA has the potential to overcome many of the 
limitations to screening large populations, as it is likely to be significantly cheaper than using echocardiography, avoids the need for highly trained health professionals, and does not require specialized healthcare facilities. This makes it attractive not only in earlier and more accurate diagnosis of asymptomatic VHD in the Western world, such as degenerative AS, but also in early detection of rheumatic valve disease, which remains the most common acquired VHD in developing countries (23). A particularly promising area appears to be the primary care setting, where VHD screening with CAA could be combined with the use of commercially available, mobile (miniaturized) echocardiography devices for triage improvement and facilitation of patient referral (14). There is a need for larger studies, validating the utility of CAA in a clinical setting in order to support its application as decision making tool in daily clinical practice.

\section{Imaging}

Echocardiography is central to every stage in the diagnosis, monitoring, and timing of intervention in all forms of VHD (24). Although widely available and relatively low cost, demand for cardiovascular imaging continues to grow exponentially and as a result, timely, unfettered access to echocardiography remains a problem in many healthcare systems. One of the major problems is the dependency of the technique on highly skilled operators to acquire images, interpret findings, and decide on subsequent management. There are several ways in which AI can improve practice in this area.

Automated measurements: ML is a technique by which AI can learn rules and identify patterns in features within datasets, which can range from pixel density, brightness, a vector of movement, or a measurement (25). Using a process such as segmentation, images or volumes can be broken down into multiple landmarks or anatomical points, such as the apex of the heart and the attachment of the aortic valve leaflets, so that over time these can be localized and tracked across frames. Segmentation and modelling can then be used in a number of ways, for example to automate measurements of 2D dimensions or of Doppler velocities, in such a way that multiple assessments can be made with greater reproducibility at a faster speed, thereby also improving efficiency (26). Accurate, serial analysis of chamber dimensions and velocities is central to management of a patient with VHD, as these are major triggers for intervention but segmentation and modelling can also be used to plan intervention, for example by producing anatomically detailed images of valves prior to minimally invasive surgery (27).

Image quality, training and analysis: The rapid development and low cost of hand-held echocardiography devices (Figure 3), combined with the widespread availability of broadband technology, has opened new opportunities for screening and community diagnosis. Although many hand-held machines offer only 2D imaging, in appropriately trained individuals, this is sufficient for accurate diagnosis of significant VHD (29). That appropriate level of training does not have to involve person-to-person contact, but can also be done at a distance with engaged but unskilled individuals with broadband support-teaching 'on the job' but at a distance (30). Once images have been acquired, communication of that data to experts directing management can accelerate appropriate intervention in VHD in rural and under-privileged communities (31). Deep learning algorithms offer the opportunity to remove some of the need for highly trained individuals to do the analysis, for example with automated analysis of chamber volumes and function (32). Fully automated interpretation of echocardiography is on the horizon, with accurate image identification and segmentation, leading to quantification of volumes and function equivalent to accredited echocardiographers (33). These deep learning algorithms currently may operate on mainframe echo machines but over time, offer opportunities to train (advising the trainee when an echo view is 'correctly aligned'), analyse (automated analysis of LV chamber dimensions), and potentially combine data to advise on risk (taking data from the images, together with data packaged from age, gender, and patient identifier).

Result communication: Despite recognition of the importance of timely diagnosis and appropriate treatment of VHD, there continues to be substantial variation in the treatment rates of patients-in European tertiary care hospitals, a quarter of patients with severe AS are not referred appropriately for intervention (3). The implications of a missed or delayed diagnosis, or inappropriate treatment, are as significant in AS, however, as they are in many forms of cancer. This has led to calls for automated alerts from echocardiography, sent directly to the requesting physician or care provider, not only highlighting the diagnosis but also outlining standards for management (34). Such alerts do not have to be generated from within the report itself by the echocardiographer but there is now capability for automated e-mail alerts to be sent direct from the machine 

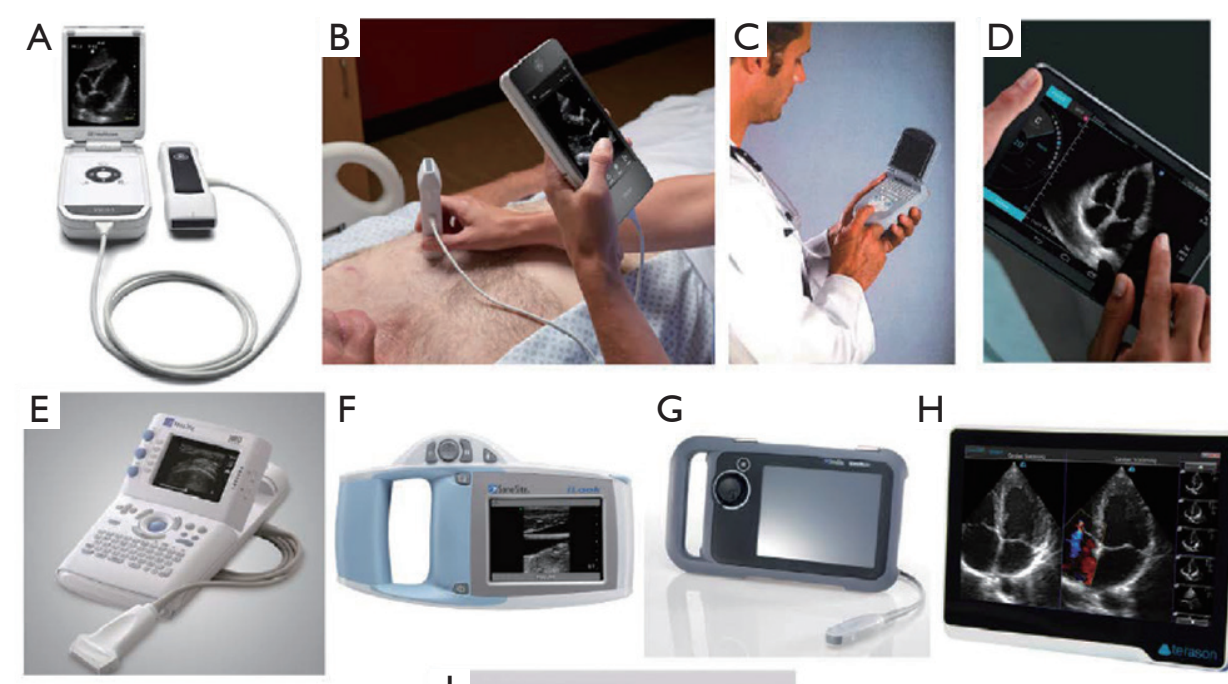

G

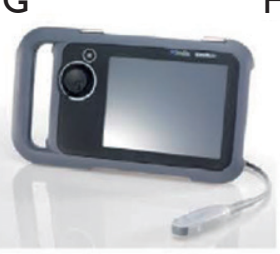

$\mathrm{H}$

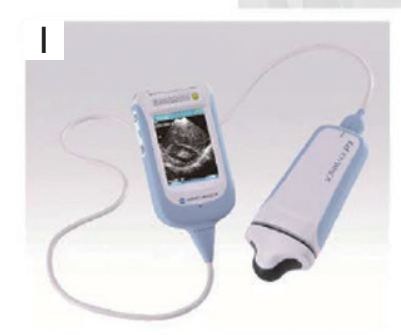

Figure 3 All-in-one handheld echocardiogram devices with electronic transducers (28). Vscan with dual probe (A) and Vscan Extend (B) (Image courtesy of GE Healthcare, Wauwatosa, WI). (C) Acuson P10, OSiemens Healthineers 2017 (used with permission), Siemens Medical Solutions Inc, Malvern, PA. (D) Iviz. (E) 180 Plus. (F) iLook. (G) Nano-Maxx (D-G Images courtesy of FUJIFILM SonoSite Inc, Bothell, WA). (H) uSmart 3200T (Image courtesy of Terason Division, Teratech Corporation, Burlington, MA). (I) Sonimage P3 (used with permission), Konica Minolta Healthcare Americas, Inc, Wayne, NJ.

on measurement when wi-fi enabled. Furthermore, there is evidence that such forms of facilitated data relay can improve timeliness of patient treatment $(3,35)$.

\section{Clustering of data for diagnosis and intervention}

Cluster analysis is a ML technique that provides a process for the creation of homogeneous groups or phenotypes from hidden patterns in data. This can be a useful tool for connecting links between apparently unrelated clinical and imaging data from electronic medical records, for example in identifying particular patterns of ventricular remodelling that carries adverse prognosis in diabetic patients (36). This has potential in a number of ways in VHD, for example by selecting outpatients at particular risk of VHD, so as to reduce the numbers needed for screening, or by selecting out those at particular risk for progression. In AS for example, it is known that risk of progression increases with clinical factors including age, diabetes mellitus, and hypertension, as well as fall in estimated glomerular filtration rate, while risk of events increases with 12-lead electrocardiogram features, such as LV hypertrophy, biomarker elevation in $\mathrm{N}$-terminal proB natriuretic peptide, and high sensitivity troponin, as well as imaging features including severity of calcification on computed tomography and presence of late gadolinium enhancement on cardiovascular magnetic resonance imaging. Interrogation of electronic medical records to identify those patients with clusters of clinical, biomarker, and imaging data will not only select individuals at high risk but may identify potentially new, unknown factors. Such tools do not have to be limited by cross-sectional analysis but modelling based on serial assessment has even greater potential to estimate trajectory of AS progression, for example by tracking changes in gradients over time, so that patient supervision and management can be individualized (37). 


\section{Treatment planning}

Aortic valve replacement by surgery or the transcatheter approach is the treatment of choice for patients with severe AS. As recommended by guidelines, the most appropriate treatment option should be determined by a multi-disciplinary heart team (MDT) and usually consider factors such as patient comorbidities, age, frailty, level of independence, anatomical conditions, peri-operative mortality risk but also patient preferences (24). Whereas valve type selection and sizing are mostly enabled by intra-operative in-situ probing in surgery, imaging (echocardiography/computed tomography $[\mathrm{CT}]$ ) plays a key role in procedure planning for transcathether valve therapies. AI-enabled clinical decision support systems (CDSSs) have been shown to support procedure-planning by integrating relevant anatomical information from echocardiography/CT into algorithms that help to determine the appropriate valve size (or even valve type) for aortic or mitral valve interventions in a fast, accurate and reliable manner (38-41). Such algorithms could be a valuable resource for high volume implanting sites, as imaging analysis is usually time-consuming, performed as a manual task, and, therefore, associated with a significant inter-operator variability.

\section{Conclusions}

AI-enabled applications, such as CAA, cardiovascular imaging (echocardiography/CT), or cluster-analysis enhanced by ML algorithms have been developed and are being increasingly introduced into clinical practice. These applications have the potential to not only support timely diagnosis-making and patient referral, but also treatment planning of patients with VHD. While AI-enabled tools are mainly targeting VHD as an underdiagnosed and undertreated condition, their systematic introduction could lead to the identification of a large number of VHD patients at all clinical stages and therefore an increasing burden to health care systems.

\section{Acknowledgments}

Funding: None.

\section{Footnote}

Reporting Checklist: The authors have completed the Narrative Review reporting checklist. Available at http:// dx.doi.org/10.21037/jtd-20-1837

Conflicts of Interest: All authors have completed the ICMJE uniform disclosure form (available at http://dx.doi. org/10.21037/jtd-20-1837). M Thoenes reports other from Edwards Lifesciences, outside the submitted work. P Bramlage reports grants and personal fees from Edwards Lifesciences, outside the submitted work. RP Steeds reports grants and personal fees from Edwards Lifesciences, outside the submitted work. The other authors have no conflict of interest to declare.

Ethical Statement: The authors are accountable for all aspects of the work in ensuring that questions related to the accuracy or integrity of any part of the work are appropriately investigated and resolved.

Open Access Statement: This is an Open Access article distributed in accordance with the Creative Commons Attribution-NonCommercial-NoDerivs 4.0 International License (CC BY-NC-ND 4.0), which permits the noncommercial replication and distribution of the article with the strict proviso that no changes or edits are made and the original work is properly cited (including links to both the formal publication through the relevant DOI and the license). See: https://creativecommons.org/licenses/by-nc-nd/4.0/.

\section{References}

1. Boudoulas KD, Borer JS, Boudoulas H. Etiology of valvular heart disease in the 21st century. Cardiology 2013;126:139-52.

2. Moore M, Chen J, Mallow PJ, et al. The direct health-care burden of valvular heart disease: evidence from US national survey data. Clinicoecon Outcomes Res 2016;8:613-27.

3. Frey N, Steeds RP, Rudolph TK, et al. Symptoms, disease severity and treatment of adults with a new diagnosis of severe aortic stenosis. Heart 2019;105:1709-16.

4. Iung B, Delgado V, Rosenhek R, et al. Contemporary Presentation and Management of Valvular Heart Disease: The EURObservational Research Programme Valvular Heart Disease II Survey. Circulation 2019;140:1156-69.

5. Davenport T, Kalakota R. The potential for artificial intelligence in healthcare. Future Healthc J 2019;6:94-8.

6. Miller DD, Brown EW. Artificial Intelligence in Medical Practice: The Question to the Answer? Am J Med 2018;131:129-33. 
7. Krittanawong C, Zhang H, Wang Z, et al. Artificial Intelligence in Precision Cardiovascular Medicine. J Am Coll Cardiol 2017;69:2657-64.

8. Kwon JM, Kim KH, Akkus Z, et al. Artificial intelligence for detecting mitral regurgitation using electrocardiography. J Electrocardiol 2020;59:151-7.

9. Jeganathan J, Knio Z, Amador Y, et al. Artificial intelligence in mitral valve analysis. Ann Card Anaesth 2017;20:129-34.

10. Fatima H, Mahmood F, Sehgal S, et al. Artificial Intelligence for Dynamic Echocardiographic Tricuspid Valve Analysis: A New Tool in Echocardiography. J Cardiothorac Vasc Anesth 2020;34:2703-6.

11. Iung B, Vahanian A. Degenerative calcific aortic stenosis: a natural history. Heart 2012;98 Suppl 4:iv7-13.

12. Frank S, Johnson A, Ross J Jr. Natural history of valvular aortic stenosis. Br Heart J 1973;35:41-6.

13. Mangione S, Nieman LZ. Cardiac auscultatory skills of internal medicine and family practice trainees. A comparison of diagnostic proficiency. JAMA 1997;278:717-22.

14. Thoenes M, Bramlage P, Zamorano P, et al. Patient screening for early detection of aortic stenosis (AS)-review of current practice and future perspectives. J Thorac Dis 2018;10:5584-94.

15. Gardezi SKM, Myerson SG, Chambers J, et al. Cardiac auscultation poorly predicts the presence of valvular heart disease in asymptomatic primary care patients. Heart 2018;104:1832-5.

16. Leng S, Tan RS, Chai KT, et al. The electronic stethoscope. Biomed Eng Online 2015;14:66.

17. Liu C, Springer D, Li Q, et al. An open access database for the evaluation of heart sound algorithms. Physiol Meas 2016;37:2181-213.

18. Clifford GD, Liu C, Moody B, et al. Recent advances in heart sound analysis. Physiol Meas 2017;38:E10-25.

19. Thompson WR, Reinisch AJ, Unterberger MJ, et al. Artificial Intelligence-Assisted Auscultation of Heart Murmurs: Validation by Virtual Clinical Trial. Pediatr Cardiol 2019;40:623-9.

20. Lai LS, Redington AN, Reinisch AJ, et al. Computerized Automatic Diagnosis of Innocent and Pathologic Murmurs in Pediatrics: A Pilot Study. Congenit Heart Dis 2016;11:386-95.

21. Grgic-Mustafic R, Baik-Schneditz N, Schwaberger B, et al. Novel algorithm to screen for heart murmurs using computer-aided auscultation in neonates: a prospective single center pilot observational study. Minerva Pediatr
2019;71:221-8.

22. Watrous RL, Thompson WR, Ackerman SJ. The impact of computer-assisted auscultation on physician referrals of asymptomatic patients with heart murmurs. Clin Cardiol 2008;31:79-83.

23. Engel M, Zühlke L, Robertson KA. ASAP programme: Rheumatic fever and rheumatic heart disease: Where are we now in South Africa? S Afr Heart J 2009;6:270-3.

24. Baumgartner H, Falk V, Bax JJ, et al. 2017 ESC/EACTS Guidelines for the management of valvular heart disease. Eur Heart J 2017;38:2739-91.

25. Dey D, Slomka PJ, Leeson P, et al. Artificial Intelligence in Cardiovascular Imaging: JACC State-of-the-Art Review. J Am Coll Cardiol 2019;73:1317-35.

26. Zolgharni M, Dhutia NM, Cole GD, et al. Automated aortic Doppler flow tracing for reproducible research and clinical measurements. IEEE Trans Med Imaging 2014;33:1071-82.

27. Ionasec RI, Voigt I, Georgescu B, et al. Patient-specific modeling and quantification of the aortic and mitral valves from 4-D cardiac CT and TEE. IEEE Trans Med Imaging 2010;29:1636-51.

28. Chamsi-Pasha MA, Sengupta PP, Zoghbi WA. Handheld Echocardiography: Current State and Future Perspectives. Circulation 2017;136:2178-88.

29. Abe $Y$, Ito $M$, Tanaka $C$, et al. A novel and simple method using pocket-sized echocardiography to screen for aortic stenosis. J Am Soc Echocardiogr 2013;26:589-96.

30. Bansal M, Singh S, Maheshwari P, et al. Value of interactive scanning for improving the outcome of new-learners in transcontinental tele-echocardiography (VISION-in-Tele-Echo) study. J Am Soc Echocardiogr 2015;28:75-87.

31. Bhavnani SP, Sola S, Adams D, et al. A Randomized Trial of Pocket-Echocardiography Integrated Mobile Health Device Assessments in Modern Structural Heart Disease Clinics. JACC Cardiovasc Imaging 2018;11:546-57.

32. Medvedofsky D, Mor-Avi V, Amzulescu M, et al. Three-dimensional echocardiographic quantification of the left-heart chambers using an automated adaptive analytics algorithm: multicentre validation study. Eur Heart J Cardiovasc Imaging 2018;19:47-58.

33. Zhang J, Gajjala S, Agrawal P, et al. Fully Automated Echocardiogram Interpretation in Clinical Practice. Circulation 2018;138:1623-35.

34. Barnhart GR, Martin RP, Thomas JD, et al. The Need for Echocardiography Alerts for Aortic Stenosis: The Time Has Come. J Am Soc Echocardiogr 2020;33:355-7. 
35. Kirby AM, Kruger B, Jain R, et al. Using Clinical Decision Support to Improve Referral Rates in Severe Symptomatic Aortic Stenosis: A Quality Improvement Initiative. Comput Inform Nurs 2018;36:525-9.

36. Ernande L, Audureau E, Jellis CL, et al. Clinical Implications of Echocardiographic Phenotypes of Patients With Diabetes Mellitus. J Am Coll Cardiol 2017;70:1704-16.

37. Kim D, Chae D, Shim CY, et al. Predicting Disease Progression in Patients with Bicuspid Aortic Stenosis Using Mathematical Modeling. J Clin Med 2019;8:1302.

38. de Jaegere P, Rocatello G, Prendergast BD, et al. Patient-specific computer simulation for transcatheter

Cite this article as: Thoenes M, Agarwal A, Grundmann D, Ferrero C, McDonald A, Bramlage P, Steeds RP. Narrative review of the role of artificial intelligence to improve aortic valve disease management. J Thorac Dis 2021;13(1):396-404. doi: $10.21037 /$ jtd-20-1837 cardiac interventions: what a clinician needs to know. Heart 2019;105:s21-7.

39. Queiros S, Dubois C, Morais P, et al. Automatic 3D aortic annulus sizing by computed tomography in the planning of transcatheter aortic valve implantation. J Cardiovasc Comput Tomogr 2017;11:25-32.

40. Astudillo P, Mortier P, Bosmans J, et al. Enabling Automated Device Size Selection for Transcatheter Aortic Valve Implantation. J Interv Cardiol 2019;2019:3591314.

41. Rosler AM, Fraportti J, Nectoux P, et al. Development and Application of a System Based on Artificial Intelligence for Transcatheter Aortic Prosthesis Selection. Braz J Cardiovasc Surg 2018;33:391-7. 